\title{
Graph-Theoretical Dynamic User Pairing for Downlink NOMA Systems
}

\author{
Alper Köse ${ }^{\circledR}$, Student Member, IEEE, Mutlu Koca ${ }^{\circledR}$, Senior Member, IEEE, \\ Emin Anarım ${ }^{\circledR}$, Muriel Médard ${ }^{\circledR}$, Fellow, IEEE, and Hakan Gökcesu ${ }^{\circledR}$
}

\begin{abstract}
We propose a novel graph-theoretical dynamic user pairing strategy based on the user rate requirements in cellular networks employing non-orthogonal multiple access (NOMA). The proposed approach relies on first constructing a conflict graph corresponding to all possible user pairings and then reformulating the problem of finding the best user pairs as that of finding the maximum weighted independent set (MWIS) on the conflict graph. This formulation turns the originally NP-hard problem into one that can be solvable in polynomial time thanks to the claw-freeness property of the conflict graph. The proposed user pairing method satisfies the maximum number of user demands with optimal network sum-rate as shown theoretically and as validated by the simulation results.
\end{abstract}

Index Terms-Non-orthogonal multiple access, user pairing, conflict graph, maximum weighted independent set.

\section{INTRODUCTION}

$\mathbf{N}$ ON-ORTHOGONAL multiple access (NOMA) has been proposed as an enabling technology to realize the data rate requirements envisioned for the next generation networks [1]. Unlike orthogonal multiple access (OMA), where the resources are orthogonally allocated to each user, NOMA principle relies on allocating multiple user signals on each resource block at the transmitter side which are decoded by using successive interference cancellation (SIC) at the receiver [1]. Since the number of supported users is not limited by the number of available orthogonal resources in NOMA, a higher number of connections, a higher system throughput and a higher spectral efficiency requirements for fifth generation $(5 \mathrm{G})$ cellular networks and beyond can be achieved by NOMA where long-term evolution (LTE) standards, which adopt orthogonal frequency-division multiple access (OFDMA), are rapidly becoming obsolete to satisfy such demands as discussed in [2] and [3].

A variety of techniques such as the use of different codebooks, waveforms, modulation types or power levels have been used to differentiate the superimposed user signals from each other on each resource block as summarized in [1] and [4]. Among these approaches, power-domain NOMA (PD-NOMA)

Manuscript received July 10, 2021; accepted July 28, 2021. Date of publication August 3, 2021; date of current version October 11, 2021. This work was supported by The Scientific and Technological Research Council of Turkey (TUBITAK) under Project 119N154. The associate editor coordinating the review of this letter and approving it for publication was P. Yang. (Corresponding author: Alper Köse.)

Alper Köse, Mutlu Koca, and Emin Anarım are with Electrical and Electronics Engineering Department, Bogazici University, 34342 Istanbul, Turkey (e-mail: alper.kose@boun.edu.tr; mutlu.koca@boun.edu.tr; anarim@ boun.edu.tr)

Muriel Médard is with the Research Laboratory of Electronics (RLE), Electrical Engineering and Computer Science (EECS), Massachusetts Institute of Technology, Cambridge, MA 02139 USA (e-mail: medard@mit.edu).

Hakan Gökcesu is with Electrical and Electronics Engineering Department, Bilkent University, 06800 Ankara, Turkey (e-mail: hgokcesu@ ee.bilkent.edu.tr).

Digital Object Identifier 10.1109/LCOMM.2021.3102116 has attracted the most attention where in the two-user case, a strong signal from/to one user and a weak signal from/to another user are assigned to the same resource block and at the receiver, the received messages are decoded in an order depending on the channel gains by employing SIC as explained in [1]. Therefore, a proper user clustering/pairing is a key factor for NOMA in order to reach its potential gains by surpassing a possible drawback which may be caused by SIC issues. The importance of user pairing to satisfy the needs of key performance indicators (KPI), such as system throughput and spectral efficiency, is shown both theoretically and numerically in [5]-[7]. In works such as [6], [8]-[12], and [13], user clustering and power allocation problems are jointly addressed for PD-NOMA. However, due to the NP-hard complexity caused by the underlying combinatorial nature of the problem, in most cases only sub-optimal solutions are proposed by simplifying the objective functions and/or by relaxing some of the original constraints. In some works, the problem requires complex user pairing and power allocation strategies which are not easily applicable in practical systems. Regarding that complex power allocation strategies would not be feasible in many practical deployment scenarios, in [10], only the user clustering/pairing optimization is addressed by adopting a predefined power allocation scheme where heuristic solutions are proposed again for the original problem. Adopting the same principle and also applying a pre-defined power allocation policy where each user is allocated a power that is proportional to the experienced path-loss, the main contribution of this letter is a graph-theoretical user-pairing strategy that is shown to be optimal in the sum-rate. The proposed algorithm circumvents the NP-hard complexity of the original problem and provides optimal sum-rate for the adopted power allocation scheme.

Specifically, in this letter, we consider a downlink communication system employing PD-NOMA with two users in each resource block and with a practical pre-defined power allocation scheme and variable user rate requirements. Two-user NOMA systems have lower decoding complexity and delay at the receivers than those in multi-user NOMA systems and therefore have more potential for applicability as noted in [7]. For the best user pairing strategy, instead of forming a conventional constrained optimization framework as in [6]-[8], [10], and [13] which will have NP-hard complexity, we take a different approach and propose a novel graphtheoretical and dynamic solution to this problem. In our approach, all possible user pairs are placed as vertices on a conflict graph and labelled with deftly specified weights. Then the problem of finding the optimal user pairs becomes that of finding the maximum weighted independent set (MWIS) on the conflict graph. Notice that unlike the NP-hard time complexity of the former problem, as presented in [14]-[16] the solution to the latter problem can be found in polynomial 
time in claw-free graphs. That is why, we first prove that the conflict graph corresponding to the NOMA user pairing problem is claw-free. Then we use this claw-free conflict graph and the graph search algorithms such as those given in [14]-[16] to determine the MWIS. The resulting MWIS provides us with the achievability of the rate demand vector of users and the pairing that guarantees to satisfy the maximum possible number of user demands in the sum-rate optimal sense. Finally, by comparing the sum-rates obtained with the proposed graph-based approach with those of the benchmark methods, we show that the proposed graph-theoretic approach clearly outperforms both the OMA setup and also the alternative user pairing optimization methods under the same channel conditions.

\section{USER PAIRING IN NOMA}

User clustering is generally an NP-hard optimization problem and all the possible combinations should be considered to find the optimal solution for any given cost function. Nevertheless, a number of heuristic solutions are also proposed to this problem such as those given in [7] and [10].

In [7], the users are paired starting with the ones which have the best and the worst channels and then with the ones having the second best and worst channels, and so on. This strategy can be written as

$$
p_{a, b}= \begin{cases}1, & \text { if } a+b=N+1 \\ 0, & \text { otherwise }\end{cases}
$$

where $p_{a, b}=1$ implies the pairing of users $a$ and $b$ assuming $\left|h_{a}\right| \geq\left|h_{b}\right|$ for $a<b$ where $a, b \in\{1,2, \ldots, N\}$.

Alternatively, the geometrical approach of [10] given for a downlink NOMA framework forms a disjoint disc and a ring. In this technique, the disc is at the center of the cell with a radius of $r_{1}$ and the ring is the difference of the cell with radius $r$ from the aforementioned disc. Then each cluster contains one user from the disc and another from the ring so as to pair a near-user that has a good channel condition with a far-user that has a worse channel condition. This approach, albeit being simple to implement, has been shown to provide satisfactory performance in [1] and [10]. An illustration for this benchmark model is provided in [10].

These approaches as well as the random pairing scheme are considered as benchmark methods used in performance comparisons with the proposed graph theoretical user pairing strategy that is presented in the following.

\section{SySTEM MODEL}

We consider a cellular downlink communication scenario where a base station (BS), located at the center of a disc having a radius $r$, serves randomly located $N$ users inside the disc. There are $\frac{N}{2}$ sub-carriers, with $N$ being an even number without loss of generality, and each sub-carrier is assigned to a pair of users. Note that we only consider the pairing scenario, so more than two users in a cluster is not allowed. We assume that each channel gain is denoted by $h_{a}=\sqrt{d_{a}^{-\gamma}} g_{a}$ where $d_{a}$ is the distance between the BS and the user $a, \gamma$ is the path-loss exponent and $g_{a}$ represents the small-scale fading coefficient which is assumed to be Rayleigh distributed. In addition, we consider a fixed power allocation scheme as in [10] and allocate powers, $q_{a}$, proportional to path-losses of users, i.e. $q_{a} \propto d_{a}^{\gamma}, \forall a$. Therefore, the power allocation coefficients are assigned as $q_{a}=\frac{d_{a}^{\gamma}}{\sum_{i=1}^{N} d_{i}^{\gamma}}, \forall a \in\{1,2, \ldots, N\}$. In this case, the signal received by user $a$, which is paired with user $b$ in the same sub-carrier, can be given as

$$
y_{a}=h_{a} \sqrt{q_{a} P_{t o t}} s_{a}+h_{a} \sqrt{q_{b} P_{t o t}} s_{b}+n_{a}
$$

where $P_{t o t}$ is the total transmit power of the BS, $s_{a}$ denotes the message sent to user $a$ and $n_{a}$ denotes the additive white Gaussian noise (AWGN) with zero mean and variance $N_{0} / 2$. We denote the bandwidth assigned to each user pair $a$ and $b$ as $B$, meaning the effective noise power experienced by each pair is $N_{0} B$.

We assume that $\left|h_{a}\right| \geq\left|h_{b}\right|$ for $a<b$ throughout this letter without any loss of generality. With this assumption, user $a$ performs SIC in order to decode the message sent to user $b$, having a poorer channel condition, and then removes this message from the overall signal to decode its own message. On the other hand, user $b$ directly decodes its own message by treating the message destined to user $a$ as noise. After the decoding operations, the data rates of users $b$ and $a$ are respectively expected to be

$$
R_{(a, b)}(b)=\log \left(1+\frac{q_{b}\left|h_{b}\right|^{2}}{q_{a}\left|h_{b}\right|^{2}+\frac{1}{S N R}}\right)
$$

and

$$
R_{(a, b)}(a)=\log \left(1+q_{a}\left|h_{a}\right|^{2} S N R\right)
$$

where $S N R=P_{t o t} / N_{0} B$ denotes the transmit signal-to-noise ratio of the BS. Notice that we can also write the rate of user $k$ in the benchmark OMA system as

$$
R(a)=\log \left(1+2 q_{a}\left|h_{a}\right|^{2} S N R\right)
$$

which corresponds to the case that user $a$ is assigned a resource block in the considered time slot with twice as much average power as that in NOMA. Finally, we denote the rate demand of user $a$ as $\bar{R}(a)$. Any user failing to reach its rate demand is considered to be in outage.

As mentioned before, a predetermined power allocation is assumed to be employed so as to maximize the fairness among users, leaving us with the problem finding the user pairing set that maximizes the number of satisfied user demands with optimal sum-rate in the network. Having the NOMA user rates in hand, the proposed optimum graph-theoretical approach for finding the optimum user pairing set is based on the construction of the so-called conflict graph that is described in the following section.

\section{The Graph-Theoretical Pairing Strategy}

We propose a novel graph-based dynamic user pairing strategy for downlink PD-NOMA that is able to achieve the maximum number of demanded rates of users in a sumrate optimal fashion. In the following, we first make some necessary definitions to describe the problem on a conflict graph, then present the construction principles of this graph and state some of the fundamental properties, which lead to the optimal user pairing solution, of the conflict graph. 


\section{A. Construction of the Conflict Graph}

Definition 1 (Conflict Graph): A graph $\mathcal{G}=(\mathcal{V}, \mathcal{E})$ is a conflict graph if there is an edge between two vertices when at most one of the variables represented by the vertices can equal one in an optimal solution [17].

Definition 2 (Claw-Free Graph): A graph $\mathcal{G}=(\mathcal{V}, \mathcal{E})$ is claw-free if none of its vertices has three pairwise nonadjacent neighbors [18]. Graphical illustration of a typical claw is not included here due to space limitations but we refer the interested reader to [14] for an example.

Definition 3 (Independent Set): Given an undirected graph $\mathcal{G}=(\mathcal{V}, \mathcal{E})$, a subset of vertices $\mathcal{S} \subseteq \mathcal{V}$ is an independent set if $\forall i, j \in \mathcal{S},\{i, j\} \notin \mathcal{E}$ is satisfied [19].

Definition 4 (Independence Number): Independence number of a graph $\mathcal{G}=(\mathcal{V}, \mathcal{E})$ is the size of the largest independent set in the graph [20].

Definition 5 (Maximum Weighted Independent Set): Given an undirected and weighted graph $\mathcal{G}=\left(\mathcal{V}, w_{\mathcal{V}}, \mathcal{E}\right)$, where the weights are assigned on vertices $w_{\mathcal{V}}: \mathcal{V} \rightarrow \mathbb{R}$, a subset of vertices $\mathcal{S} \subseteq \mathcal{V}$ constitute a maximum weighted independent set if $\forall i, j \in \mathcal{S},\{i, j\} \notin \mathcal{E}$ and there is no other independent set having a greater weight sum $\sum_{i \in \mathcal{S}} w_{i}$ [19].

The user pairing conflict graph is the graph of possible pairings among users. In the construction of the conflict graph $\mathcal{G}=\left(\mathcal{V}, w_{\mathcal{V}}, \mathcal{E}\right), \mathcal{V}$ is the set of possible pairings, $w_{\mathcal{V}}$ is the set of weights belonging to $\mathcal{V}$ and $\mathcal{E}$ is the set of conflicts. The vertices, weights and edges of the conflict graph are found as follows: Given $N$ users indexed from 1 to $N$, each vertex of the conflict graph corresponds to a pair of users $(a, b)$, where $1 \leq a<N$ and $a<b \leq N$.

The weight $w_{(a, b)}$ of the vertex of user pair $(a, b)$ is given in Eq. (5), as shown at the bottom of the page, where $R_{(a, b)}(a)$ is the data rate of user $a$ assuming that user $a$ is paired with user $b, \bar{R}(a)$ is the rate demand of user $a . \mu$ denotes the rate bias that is significantly bigger than the user rates which can be chosen as

$$
\mu=\sum_{\substack{a, b \\ 1 \leq a<N \\ a<b \leq N}}\left[R_{(a, b)}(a)+R_{(a, b)}(b)\right]
$$

and which corresponds to the sum-rate for all possible user pairs without any rate requirements. Notice that the last case in Eq. (5) corresponds to the 0 rate for both users in the pair. However, in order to avoid numerical instability problems in MWIS algorithms, we employ a small non-zero rate which is denoted by $0_{+}$. Also, the data rate contributions of users who are in outage (those unable to reach their data rate demands) are omitted in the weight assignment and rate calculations.

Introducing the edges (conflicts) to the conflict graph is straightforward. The vertex containing $(a, b)$ pair must have edges with all vertices containing $(a,$.$) and (b,$.$) pairings,$ except with itself, due to the fact that we cannot pair a user

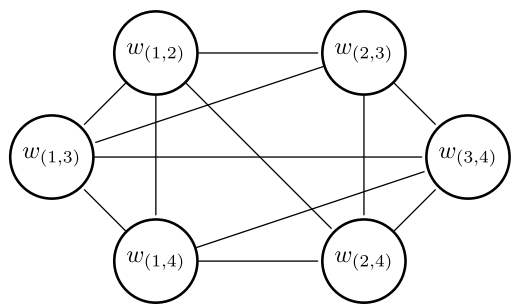

Fig. 1. User pairing conflict graph for a 4-user NOMA scheme.

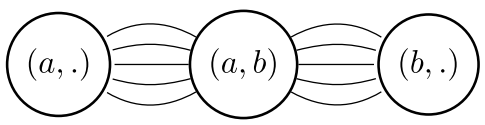

Fig. 2. Illustration for proof of Theorem 1.

with two different users simultaneously. An example of user pairing conflict graph for a 4-user NOMA scheme is shown in Fig. 1. Notice that this construction turns the original sumrate maximum user pairing problem into that of finding the MWIS over a conflict graph.

\section{B. Achievability of the Demanded User Rates}

To understand the benefits of the bias term $\mu$, suppose we are able to obtain the MWIS, $\mathcal{S} \subseteq \mathcal{V}$, of the conflict graph so that we have the total weight, $\mathcal{W}=\sum_{i \in \mathcal{S}} w_{i}$, and the vertices, $\mathcal{S}$, whose weights sum up to the weight of the MWIS are known. By choosing $\mu$ as in (6), the maximum number of users achieving their demanded rates $M$ and the resulting sum-rate in the network $\mathcal{R}$ can be found, respectively, as

$$
\begin{aligned}
M & =\mathcal{W} / \mu, \\
\mathcal{R} & =\bmod (\mathcal{W}, \mu) .
\end{aligned}
$$

Note that Eq. (7) shows an integer division. If $M=N$, we conclude that the demanded rates are achievable and the achieving pairings are given as $\mathcal{S}$. Also, the resulting pairing is sum-rate optimal after guaranteeing the satisfaction of users' rate demands. If $M<N$, the demand vector is not achievable by any pairing in the system. In this case, the graph-based pairing strategy aims at maximizing the number of successfully served users where the result is also sum-rate optimal.

\section{Properties of the NOMA User Pairing Conflict Graph}

We now present some of the key properties of the NOMA user pairing conflict graph as follows:

Theorem 1: The user pairing conflict graph is guaranteed to be claw-free.

Proof: Consider a vertex representing a possible pairing of users $a$ and $b$ as the central vertex of a possible claw in the user pairing conflict graph as in Fig. 2. Then, there must be at least 3 different vertices which are connected to the vertex $(a, b)$ but not connected to each other in order to set up a claw

$$
w_{(a, b)}= \begin{cases}2 \mu+R_{(a, b)}(a)+R_{(a, b)}(b), & \text { if } R_{(a, b)}(a) \geq \bar{R}(a), R_{(a, b)}(b) \geq \bar{R}(b) \\ \mu+R_{(a, b)}(a), & \text { if } R_{(a, b)}(a) \geq \bar{R}(a), R_{(a, b)}(b)<\bar{R}(b) \\ \mu+R_{(a, b)}(b), & \text { if } R_{(a, b)}(a)<\bar{R}(a), R_{(a, b)}(b) \geq \bar{R}(b) \\ 0_{+}, & \text {if } R_{(a, b)}(a)<\bar{R}(a), R_{(a, b)}(b)<\bar{R}(b)\end{cases}
$$


in the graph, i.e., the independence number of the vertices connected to the vertex $(a, b)$ must be at least 3 . We define $(a,$.$) as the set of possible pairings of user a$ with others, also $(b,$.$) is defined in the same manner. It is clear that the vertex$ $(a, b)$ must be connected to all vertices in the set $(a,$.$) since$ if $a$ is paired with $b$, then any other pairing is not possible for $a$. Note that there are $N-2$ such vertices in the set $(a,$.$) .$ However, since all of the vertices are connected to each other leading to a complete graph, the independence number of the set $(a,$.$) is 1$. The same logic applies to the set $(b,$.$) leading to$ a total independence number of 2 for the sub-graph of vertices connected to the central vertex $(a, b)$. A third vertex cannot be found to set up a claw. Hence, we conclude that the user pairing conflict graph is claw-free.

Notice that as shown in [14]-[16], the MWIS can be obtained over a claw-free conflict graph in polynomial time. In this regard, the outcome of Theorem 1 is significant because it implies that finding the NOMA user pairing solution is not NP-hard but can be found in polynomial time since the corresponding conflict graph is claw-free. However, in order for the MWIS formulation to work, an important constraint is to have all users paired with each other while leaving no user being unpaired, which is a condition guaranteed by the following theorem.

Theorem 2: The polynomial time MWIS algorithm implemented on a claw-free user pairing conflict graph has all users paired.

Proof: We prove the theorem by contradiction. Assume that there are $N$ users to be paired in the network, $N$ being even, without loss of generality. Firstly, consider the case that the output of the MWIS algorithm has a cardinality $|X|$ and $|X| \geq \frac{N}{2}+1$. Having $\frac{N}{2}+1$ or more vertices means having $N+2$ or more users in the final set, therefore at least 2 users have to be repeated. However, this cannot be satisfied due to the violation of the independence of the chosen set. Now, consider the case that the output of the MWIS algorithm has a cardinality $|X| \leq \frac{N}{2}-1$. Having $\frac{N}{2}-1$ or less vertices means having $N-2$ or less users in the final set, therefore at least 2 users remain unpaired. This, in turn, means that the weight of the final set can be increased by adding the vertex (pair) of missing users. Therefore, a set with cardinality $|X| \leq \frac{N}{2}-1$ cannot be the MWIS of the graph. As a result, $|X|=\frac{N}{2}$ is satisfied and no user will be unpaired.

Example I: Consider the conflict graph in Fig. 1. The independent sets of the conflict graph are \{\}$,\{(1,2)\}$, $\{(1,3)\},\{(1,4)\},\{(2,3)\},\{(2,4)\},\{(3,4)\},\{(1,2),(3,4)\}$, $\{(1,3),(2,4)\}$ and $\{(1,4),(2,3)\}$. Note that MWIS has to be the one(s) with the highest cardinality set(s), $\{(1,2),(3,4)\}$, $\{(1,3),(2,4)\}$ or $\{(1,4),(2,3)\}$ in this case, since such sets are guaranteed to have greater weight than the sets with less elements. Thanks to this fact, the set that an MWIS algorithm produces not only has the greatest sum-rate but also the one that guarantees the pairing of all users.

Corollary I: Given $N$ users and $\frac{N}{2}$ resources in a NOMA system, the optimal user pairing solution is found in polynomial time.

Proof: The user pairing conflict graph is claw-free as proven in Theorem 1, hence the MWIS can be found in polynomial time and there will not be any unpaired users in

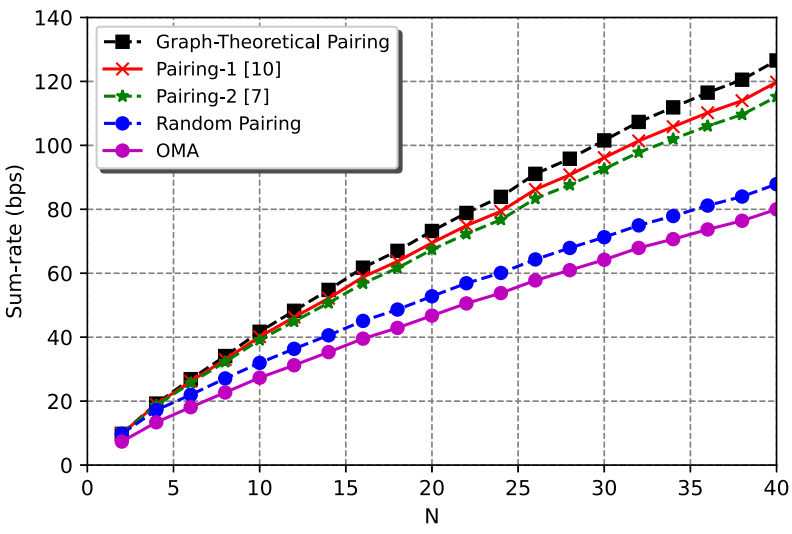

Fig. 3. Sum-rate comparison of pairing strategies with zero user rate requirements.

the end as shown in Theorem 2. Since we are able to achieve the maximum number of satisfied rate demands in a sumrate optimal manner when we found the MWIS as explained in Section IV-B, we get the optimal pairing in polynomial time.

\section{Overall Computational Complexity}

The computational complexity of creating vertices to model the possible pairings becomes $\mathcal{O}\left(N^{2}\right)$ since there are $N$ users and thus $N(N-1) / 2$ different pairings. 2 different user rates are also calculated per vertex, so the complexity order does not change due to the rate calculations; i.e., it remains $\mathcal{O}\left(N^{2}\right)$ throughout this step. Lastly, since there are $N-2$ edges in average per each possible pairing, there will be $\frac{N(N-1)(N-2)}{2}$ edges in total. In short, the construction of the conflict graph has a computational complexity of $\mathcal{O}\left(N^{3}\right)$. The complexity of the MWIS algorithm given in [16] is $\mathcal{O}(|\mathcal{V}|(|\mathcal{E}|+|\mathcal{V}| \log |\mathcal{V}|))$. Since $|\mathcal{V}|=N(N-1) / 2$ and $|\mathcal{E}|=\frac{N(N-1)(N-2)}{2}$ in the user pairing conflict graph, the MWIS algorithm has a computational complexity of $\mathcal{O}\left(N^{5}\right)$. In other words, the complexity of the MWIS algorithm clearly determines the overall computational complexity of the graph-theoretical user pairing method. This result is encouraging for small networks having moderate number of users. On the other hand, the complexity can be an issue for larger networks having large number of users. However, we observe that in such cases the graph-theoretical approach presented here can be used in conjunction with geographical clustering approaches to reduce the complexity back to manageable levels.

\section{Simulation Results and Discussion}

The simulations are conducted over $N$ users which have distances to BS chosen uniformly random between $10 \mathrm{~m}$ and $1 \mathrm{~km}$, in a cell having $r=1 \mathrm{~km}$ radius with a path-loss coefficient $\gamma=3.6$ and repeated for 500 times for each $N$ to obtain the average sum-rates. In all simulations, different number of user scenarios where $N=2-40$ are considered. A channel model in a sub-urban environment is considered and thus a path-loss exponent of 3.6 is chosen as reported in [21]. In Fig. 3, there is not any constraint on the minimum user rate requirement whereas there is a minimum demanded 


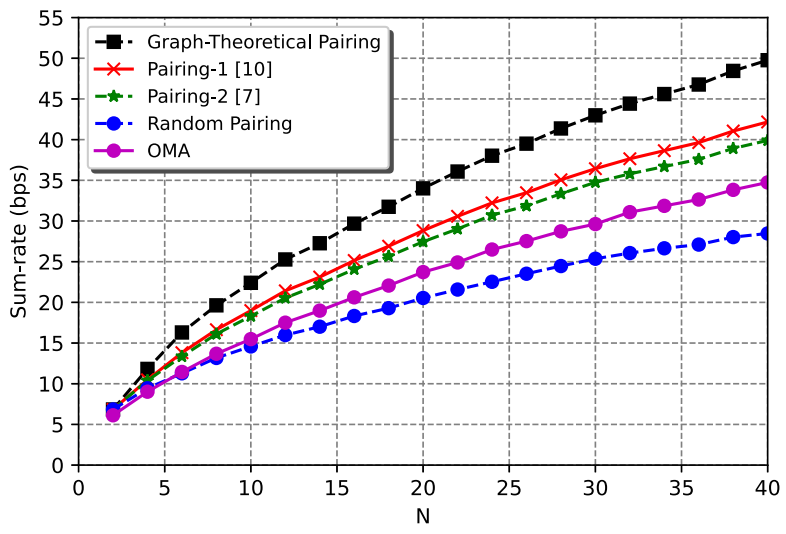

Fig. 4. Sum-rate comparison of pairing strategies with different user rate requirements.

rate chosen uniformly random between $0-10$ bps for each user in the results shown Fig. 4. We compare the sumrate performances of the standard OMA setup, NOMA with random pairing, our graph-theoretical pairing strategy adopted in NOMA, and the benchmarks obtained from some recent NOMA user pairing research letters seen in [7] and [10] and explained in Section II. In the figures, Pairing-1 and Pairing2 denote the benchmark user pairing approaches proposed in [10] and [7], respectively.

The sum-rates as a function of the number of users are shown in both figures. For $N=2$ users, all pairing strategies perform the same as expected since there is only one possible choice. However, when $N$ starts to grow, our pairing strategy starts to outperform the rival approaches thanks to optimality we can reach. In Fig. 3, since the demanded rate vector is achievable, i.e. zero demand, we only observe the performance gain which comes from the sum-rate optimality and the gain is numerically recorded as approximately $5 \%$ over the closest benchmark. These performance comparisons between pairing strategies are obtained by averaging the percentage differences for all $N \geq 20$.

In Fig. 4, due to the achievability problems in the demanded rates, the sum-rate performances are reduced in general. However, the gains obtained by the proposed strategy increases to approximately $20 \%$ over its closest counterpart ( $15 \%$ from the increased demand achievability and $5 \%$ from the sum-rate optimality). As expected, the graph-theoretical strategy acts as an upper bound for all the other strategies.

\section{CONCLUSION}

In this letter, user pairing in NOMA has been considered and a dynamic graph-theoretical solution has been proposed. We have showed that the optimal pairing in terms of the number of successfully served users and then the sum-rate can be completed in polynomial time by exploiting the claw-freeness of the user pairing conflict graph given that a power allocation scheme, equal received power in this case, is specified beforehand. We have corroborated the theoretical findings with simulation results which indicate that the graph-theoretical method has a better achievability and sum-rate performance compared to those obtained by alternative approaches.

Notice that clustering of more than two users is not considered due to the space limitations. Moreover, in this case the related conflict graph would not be claw-free and the optimality of the sum-rate maximizing user grouping would no longer be guaranteed. Nevertheless the general graph-theoretical user clustering problem and its performance compared to other clustering optimizations is considered as an interesting future research work.

\section{REFERENCES}

[1] Y. Saito, Y. Kishiyama, A. Benjebbour, T. Nakamura, A. Li, and K. Higuchi, "Non-orthogonal multiple access (NOMA) for cellular future radio access," in Proc. IEEE 77th Veh. Technol. Conf. (VTC Spring), Dresden, Germany, Jun. 2013, pp. 1-5.

[2] Z. Ding, Z. Yang, P. Fan, and H. V. Poor, "On the performance of non-orthogonal multiple access in $5 \mathrm{G}$ systems with randomly deployed users," IEEE Signal Process. Lett., vol. 21, no. 12, pp. 1501-1505, Dec. 2014.

[3] K. Yang, N. Yang, N. Ye, M. Jia, Z. Gao, and R. Fan, "Non-orthogonal multiple access: Achieving sustainable future radio access," IEEE Commun. Mag., vol. 57, no. 2, pp. 116-121, Feb. 2019.

[4] B. Kim et al., "Non-orthogonal multiple access in a downlink multiuser beamforming system," in Proc. IEEE Military Commun. Conf. (MILCOM), San Diego, CA, USA, Nov. 2013, pp. 1278-1283.

[5] Z. Ding et al., "Impact of user pairing on $5 \mathrm{G}$ nonorthogonal multipleaccess downlink transmissions," IEEE Trans. Veh. Technol., vol. 65, no. 8, pp. 6010-6023, Aug. 2016.

[6] W. Liang, Z. Ding, Y. Li, and L. Song, "User pairing for downlink nonorthogonal multiple access networks using matching algorithm," IEEE Trans. Commun., vol. 65, no. 12, pp. 5319-5332, Dec. 2017.

[7] L. Zhu, J. Zhang, Z. Xiao, X. Cao, and D. O. Wu, "Optimal user pairing for downlink non-orthogonal multiple access (NOMA)," IEEE Wireless Commun. Lett., vol. 8, no. 2, pp. 328-331, Apr. 2019.

[8] V.-P. Bui, P. X. Nguyen, H. V. Nguyen, V.-D. Nguyen, and O.-S. Shin, "Optimal user pairing for achieving rate fairness in downlink NOMA networks," in Proc. Int. Conf. Artif. Intell. Inf. Commun. (ICAIIC), Feb. 2019, pp. 575-578.

[9] J. Cui, Y. Liu, Z. Ding, P. Fan, and A. Nallanathan, "Optimal user scheduling and power allocation for millimeter wave NOMA systems," IEEE Trans. Wireless Commun., vol. 17, no. 3, pp. 1502-1517, Mar. 2017.

[10] Z. Ding, R. Schober, and H. V. Poor, "A general MIMO framework for NOMA downlink and uplink transmission based on signal alignment," IEEE Trans. Wireless Commun., vol. 15, no. 6, pp. 4438-4454, Jun. 2016.

[11] L. Zhu, J. Zhang, Z. Xiao, X. Cao, D. O. Wu, and X. Xia, "Millimeter-wave NOMA with user grouping, power allocation and hybrid beamforming," IEEE Trans. Wireless Commun., vol. 18, no. 11, pp. 5065-5079, Nov. 2019.

[12] K. Wang, J. Cui, Z. Ding, and P. Fan, "Stackelberg game for user clustering and power allocation in millimeter wave-NOMA systems," IEEE Trans. Wireless Commun., vol. 18, no. 5, pp. 2842-2857, May 2019.

[13] J. Zhu, J. Wang, Y. Huang, S. He, X. You, and L. Yang, "On optimal power allocation for downlink non-orthogonal multiple access systems," IEEE J. Sel. Areas Commun., vol. 35, no. 12, pp. 2744-2757, Dec. 2017.

[14] G. J. Minty, "On maximal independent sets of vertices in claw-free graphs," J. Combinat. Theory, B, vol. 28, no. 3, pp. 284-304, Jun. 1980.

[15] D. Nakamura and A. Tamura, "A revision of Minty's algorithm for finding a maximum weight stable set of a claw-free graph," J. Oper. Res. Soc. Jpn., vol. 44, no. 2, pp. 194-204, Jun. 2001.

[16] Y. Faenza, G. Oriolo, and G. Stauffer, "Solving the weighted stable set problem in claw-free graphs via decomposition," J. ACM, vol. 61, no. 4, pp. 1-41, Jul. 2014

[17] A. Atamtürk, G. L. Nemhauser, and M. W. P. Savelsbergh, "Conflict graphs in solving integer programming problems," Eur. J. Oper. Res., vol. 121, no. 1, pp. 40-55, Feb. 2000.

[18] M. Chudnovsky and P. D. Seymour, "The structure of claw-free graphs," Surv. Combinatorics, vol. 327, pp. 153-171, Jul. 2005.

[19] A. Köse, H. Gökcesu, N. Evirgen, K. Gökcesu, and M. Médard, "A novel method for scheduling of wireless ad hoc networks in polynomial time," IEEE Trans. Wireless Commun., vol. 20, no. 1, pp. 468-480, Jan. 2021.

[20] N. Alon and N. Kahale, "Approximating the independence number via the $\vartheta$-function," Math. Program., vol. 80, no. 3, pp. 253-264, Feb. 1998.

[21] T. S. Rappaport, Wireless Communications: Principles and Practice. New York, NY, USA: Prentice-Hall, 1998. 